\title{
Das Modell Barcelona - Partizipation, Protest und Postpolitik
}

\author{
Catarina Gomes de Matos
}

\begin{abstract}
Das ,Modell Barcelona' steht für eine Stadtplanung, bei der unterschiedliche Akteur_innen vermeintlich vorbildlich in Entscheidungs- und Entwicklungsprozesse einbezogen sind. Die heutigen formellen Beteiligungsformen werden dabei meist als in der Tradition der nachbarschaftlichen Proteste in den 1970er Jahren stehend gesehen und die zunehmende Formalisierung von Bürger_innenbeteiligung wird als Prozess der Demokratisierung verstanden. In meinem Beitrag wird der Zusammenhang zwischen partizipativen Verfahren und Demokratie grundlegend hinterfragt und dem traditionellen Demokratiebegriff ein emanzipatorisches Verständnis von Demokratie gegenübergestellt, das sich an Theorien der poststrukturalistischen politischen Philosophie orientiert. Anhand einer empirischen Fallstudie zum aktuellen Widerstand gegen den Umbau des Hafengebiets Port Vell in Barcelona wird gezeigt, inwiefern formale Partizipationsmöglichkeiten die Teilhabe der Bevölkerung nicht nur nicht unterstützen, sondern letztlich einschränken oder verhindern. Abschließend wird diskutiert, ob nicht formalisierte Proteste eine Alternative zu formellen Partizipationsverfahren darstellen können.
\end{abstract}

Ersteinreichung: 1. Mai 2013; Veröffentlichung online: 7. Dezember 2013

An english abstract can be found at the end of the document.

\section{Einleitung}

Partizipationsprogramme gelten gemeinhin als geeignete Strategie, um eine demokratischere Gesellschaft $\mathrm{zu}$ schaffen. Sie werden folglich in unterschiedlichen gesellschaftlichen Bereichen implementiert (Simons/Masschelein 2010, Alt/Teubner/Winklhofer 2005, Renner/Gohl/de Maizière 2002). Seit den 1970er Jahren gibt es diese Demokratisierungsbestrebungen auch in der Stadtplanung und zahlreiche Kommunen haben partizipative Verfahren eingeführt, die heute als Standard gelten. Eines der bekanntesten Beispiele der Bürger_innenbeteiligung, das die Fachöffentlichkeit als gelungen bewertet, ist das ,Modell Barcelona'. Es steht für einen Stadtplanungsansatz, bei dem man eine Vielzahl unterschiedlicher Akteur_innen in Entscheidungs- und Entwicklungsprozesse einbezieht. Dabei werden partizipative Verfahren mit dem Argument, die Stadtplanung demokratischer gestalten zu wollen, zunehmend erweitert und formalisiert. Diese formalen Partizipationsprozesse werden heute als positives Ergebnis der zivilgesellschaftlichen Proteste in den 1970er Jahren dargestellt. Zwar gibt es durchaus Stimmen, die sich kritisch gegenüber dem ,Modell Barcelona’ äußern, indem sie auf Umsetzungsdefizite verweisen. 
Das Verständnis von Partizipation, das dem Ansatz in Barcelona zugrunde liegt, wird jedoch auch von den Kritiker_innen kaum hinterfragt.

Der vorliegende Beitrag verfolgt das Ziel, den oftmals als selbstverständlich angenommenen Zusammenhang von Demokratie und partizipativen Verfahren infrage zu stellen. Dazu wird im ersten Teil dem klassischen Demokratiebegriff ein alternatives emanzipatorisches Verständnis von Demokratie gegenübergestellt. Dieses stützt sich auf die theoretischen Ansätze einer poststrukturalistischen politischen Philosophie und insbesondere auf die Arbeiten von Jacques Rancière und Chantal Mouffe. Durch diese Redefinition des Demokratiebegriffs wird die Möglichkeit geschaffen, das ,Modell Barcelona' und die Rolle zivilgesellschaftlicher Widerstände neu zu denken. Im zweiten Teil des Beitrags wird die hegemoniale Darstellung der Stadtentwicklung Barcelonas als eine Geschichte der kontinuierlichen Erweiterung von Partizipationsmöglichkeiten seit dem Ende der Diktatur Francos rekonstruiert und hinterfragt. Vor dem Hintergrund eines emanzipatorischen Demokratieverständnisses wird herausgearbeitet, wie sich die Optionen der Bürger_innenbeteiligung im zeitlichen Verlauf qualitativ verändert haben. Im dritten Teil des Beitrags werden am Beispiel des Konflikts um die Neugestaltung des innerstädtischen Hafengebiets Port Vell in Barcelona [1] zudem die Grenzen der seit den 1990er Jahren vorherrschenden Partizipationsformen aufgezeigt. Weiterhin wird die Frage behandelt, inwiefern Proteste den Anwohner_innen im Unterschied zu den behandelten partizipativen Verfahren eine Möglichkeit bieten, ihre Forderungen nach einer, echten Demokratie' zu artikulieren.

Im Folgenden wird in diesem Text von drei unterschiedlichen Demokratiebegriffen die Rede sein. Es wird unterschieden zwischen erstens einem traditionellen Verständnis von Demokratie (,traditionelle Demokratie'), zweitens einer emanzipatorischen Auffassung von Demokratie in Anschluss an Rancière und Mouffe (,emanzipatorische Demokratie') und drittens der Idealvorstellung und Forderung der Gegner_ innen der Neugestaltung des Port Vell (,echte Demokratie'). Der Begriff der Partizipation wird in Anschluss an Bonet i Martí (2012: 22) als „das Einbeziehen der Bevölkerung [...] als Akteure in die Entscheidungsprozesse während einer oder mehrerer Phasen (Diagnose, Entwurf, Implementierung, Evaluation) der Entwicklung öffentlicher Politiken“ (Übers. d. A.) definiert und unterteilt in Partizipation ,von oben' und Partizipation ,von unten'. Unter dem ersten Begriff werden partizipative Verfahren gefasst, die von den politischen Autoritäten ausgehen (participación por invitación - Teilhabe durch Einladung), unter dem zweiten solche, die von der Bevölkerung initiiert sind (participación por irrupción - Teilhabe durch Ausbruch).[2] Letztere sind meist dadurch gekennzeichnet, dass bestehende politische Partizipationskanäle unterwandert bzw. informelle Kanäle oder Mittel genutzt werden (z.B. Proteste oder Besetzungen), um auf die Stadtplanung Einfluss zu nehmen.

\section{Welche Demokratie?}

In den Politikwissenschaften wird Demokratie meist als Regierungsform oder als Modus des gesellschaftlichen Zusammenlebens definiert. Diese 
traditionelle Vorstellung von Demokratie kann als „Regierungsform, die aus dem Volk hervorgeht und durch das Volk in seinem ureigenen Interesse ausgeübt wird" (Schmidt 2000: 22), verstanden werden oder lehnt sich zumindest an eine solche Vorstellung der Herrschaft des Volkes an. Ein zentrales Kriterium solcher Definitionen von Demokratie ist also die Entscheidungsmacht oder -teilhabe der Bevölkerung. Diese kann in ihrer realen Umsetzung sehr verschieden ausgestaltet sein. Colin Crouch (2008: 9ff.) zeigt auf, welche unterschiedlichen Formen von Teilhabe der Bevölkerung unter dem Begriff der Demokratie gefasst werden: Die Spannbreite reicht von einer eher passiven Rolle der Bevölkerung in der ,liberalen Demokratie' (in der sie vorgegebenen Alternativen zustimmen oder diese ablehnen kann) über die Möglichkeit, sich an mehr oder weniger freien Wahlen zu beteiligen, bis hin zur Vorstellung von einer ,idealen Demokratie', charakterisiert durch eine aktive Gestaltung des öffentlichen Lebens (im Austausch und im Rahmen unabhängiger Organisationen). Die Gemeinsamkeit all dieser Vorstellungen ist, dass das Volk - bereits vor einer eigenen politischen Intervention - als politisches Subjekt bestimmt und definiert ist.

Diesem klassischen Demokratieverständnis steht in der Tradition der poststrukturalistischen politischen Philosophie ein alternatives, emanzipatorisches Verständnis von Demokratie gegenüber. Ein zentraler Aspekt dieser Ansätze ist die Unterscheidung zwischen ,Polizei“ bzw. ,Politik ${ }^{6}$ als Bezeichnungen für die vorherrschende soziale Ordnung auf der einen und dem ,Politischen“ als Unterbrechung dieser Ordnung auf der anderen Seite. Oliver Marchart (2010: 180) benennt diese Unterscheidung als ,politische Differenz'. Grundlegend für eine solche Unterscheidung ist das Verständnis der aktuellen sozialen Ordnung als „symbolische Konstitution des Sozialen“ (Rancière 2008: 31) - und damit als nur eine mögliche Ordnung unter vielen -, die jedoch im Alltag als ,natürliche Ordnung' erscheint (Rancière 1997: 102). Sie wird durch ein Ensemble aus Praktiken und Institutionen organisiert und aufrechterhalten (Krasmann 2010: 78, Marchart 2010: 41). Dieses Ensemble bezeichnet Jacques Rancière (2008: 31) als Polizei. Chantal Mouffe (2005: 9) verwendet in ähnlicher Weise den Begriff der Politik. Zu den Elementen dieses Ensembles zählen nicht nur die im Alltag unter dem Begriff Politik zusammengefassten politischen Systeme oder politischen Institutionen, sondern beispielsweise auch Regierungsweisen und -techniken. Auch die von Michel Foucault konzeptionalisierten und beschriebenen Technologien und Strategien der Macht fasst Rancière unter den Begriff der Polizei (Simons/Masschelein 2010: 591).

Im Rahmen dieser polizeilichen Ordnung werden den Individuen in der Gesellschaft unterschiedliche Subjektpositionen zugewiesen. Dadurch, dass bestimmte Subjektpositionen verunmöglicht und bestimmte Formen des Sprechens als bloßer ,Lärm ' unhörbar gemacht werden, wird auch festgelegt, wer an der Gesellschaft teilhaben kann und wer nicht (Krasmann 2010: 78, Rancière 2002: 41). Die gesellschaftliche Wahrnehmung darüber, wie die geeignete soziale Ordnung auszusehen hat, wird im jeweils hegemonialen Diskurs geformt. Die polizeiliche Ordnung stellt sich dabei selbst als Verwirklichung der dieser Gesellschaft zugrunde liegenden gemeinschaftlich geteilten Fundamente (Simons/Masschelein 2010: 592) und als 
einzige (,natürliche') gesellschaftliche Ordnung dar. Sie sichert ihr eigenes Fortbestehen, indem alles, was außerhalb dieser Ordnung steht, verleugnet wird (Krasmann 2010: 78). Infolgedessen wird die Grenze zwischen innen und außen unsichtbar (Simons/Masschelein 2010: 592).

Das Politische ist hingegen die Unterbrechung der aktuellen Ordnung. Es ist weder über seinen Ort oder Gegenstand noch durch seine Akteure definiert, sondern durch seine Form: durch den Zusammenstoß zweier unterschiedlicher Vorstellungen von gesellschaftlicher Aufteilung (Rancière 2008: 37). Das Politische „setzt erst in dem Moment ein, in dem die etablierten Regeln und Grenzen politischer Teilhabe zur Disposition stehen“ (Krasmann 2010: 77). Indem die Welt, die sich in der herrschenden polizeilichen Ordnung als die Einzige darstellt und außer der es nichts zu geben scheint, mit etwas konfrontiert wird, was außerhalb dieser liegt einem „Supplement“ (Rancière 2008: 24) -, wird es möglich, die bis dahin unsichtbare Grenze aufzuzeigen und zu verschieben zwischen dem, was Teil der Gesellschaft ist, und dem, was als Außen nicht dazugehört und nicht gesehen wird (ebd.). Politische Interventionen beschränken sich also nicht darauf, Änderungen innerhalb des als gegeben angesehenen Rahmens durchzusetzen. Vielmehr versuchen sie den Rahmen zu verändern, „der festlegt, wie die Dinge funktionieren“ (Žižek 2010: 273). Sie sind dabei zum einen immer eine Darstellung der , anderen' Welt, aus der sie stammen (Rancière 2008: 36). Zum anderen sind sie stets auch ein Kampf darum, dass die eigene Stimme ,als die eines rechtmäßigen Partners gehört und anerkannt" wird (Žižek 2010: 256). Spricht man vor diesem theoretischen Hintergrund von emanzipatorischer Demokratie, verweist der Begriff auf das Aufrechterhalten von Streit über die Form des gesellschaftlichen Zusammenlebens; ein Streit, der über reine Interessenskonflikte hinausgeht und die Grenzen der gesellschaftlichen Ordnung tangiert. Emanzipatorische Demokratie stellt somit immer eine Gefahr für die hegemoniale Ordnung dar (Simons/Masschelein 2010: 589).

Das Politische kann immer nur ,punktuell' und per se nicht dauerhaft sein. Es zeigt sich in den Momenten, in denen konkurrierende Kräfte interagieren und um eine neue gesellschaftliche Ordnung kämpfen. In diesen Momenten wird die scheinbar kohärente Anordnung von Orten, Funktionen und Seinsweisen umgedeutet und hinterfragt (Marchart 2010: 335). Dabei wird die Existenz eines politischen Subjekts nicht als Voraussetzung für eine politische Interaktion gesehen, sondern es konstituiert sich als solches im Moment der politischen Intervention. Häufig wird der Demokratie generell vorgeworfen, nicht mehr, richtig zu funktionieren' und defizitär zu sein. Geht man jedoch von einem emanzipatorischen Konzept von Demokratie als Subjektivierung des Politischen aus, dann ist Demokratie allerdings nicht „konjunkturell defizitär”, sondern „konstitutiv defizitär”, „,weil sie die Unmöglichkeit einer politischen Letztbegründung und damit die Kontingenz jedes Gemeinwesens in ihre institutionelle Form integriert, weshalb [...] das Unbehagen an der Demokratie zur Demokratie selbst gehört [...]" (Marchart 2010: 337). Die „in letzter Instanz unmögliche Institution von Gesellschaft" (ebd.: 27) liegt ebenso in ihrer Natur wie das Streben nach danach. 
Mit den Begriffen der Postdemokratie (Crouch 2008, Rancière 1997 und 2008) und der Postpolitik [3] (Žižek 2010, Mouffe 2005) als Zustandsbeschreibung der aktuellen Gesellschaft verweisen unterschiedliche Theoretiker_innen auf einen aus ihrer Sicht bestehenden Mangel an Möglichkeiten, politisch zu intervenieren. Mouffe (2005: 13) beschreibt die aktuelle postpolitische Gesellschaft als eine, in der die Marktlogik auf den Bereich der Politik übertragen wird. Personen werden dementsprechend als rationale Wesen gesehen, die immer ihren größtmöglichen Nutzen anstreben. In der Konsequenz wird jeder Dissens als Konflikt zwischen Konkurrent_innen definiert, die unterschiedliche Interessen verfolgen. Ein ,rationaler Konsens' wird als reale und einzige Möglichkeit gesehen, solche Interessenkonflikte aufzulösen und - indem gegensätzliche Interessen „rational versöhnt“" (ebd.: 21) werden - den Dissens zwischen den unterschiedlichen Akteur_innen zu überwinden. Verstanden wird dieser rationale Konsens als eine Win-win-Situation, eine Lösung, bei der alle Beteiligten profitieren (ebd.: 5f.). Erreicht werden soll er durch Interaktion und eine Aushandlung der Interessen aller auf der Grundlage freier Meinungsäußerung und unterstützt durch Expert_innenwissen (Žižek 2010: 272f.).

Ein solcher Konsens ist dabei als die Herstellung eines weitgehend anerkannten Kompromisses zu verstehen, der auf einer vermeintlich gemeinsamen gesellschaftlichen Grundlage an Werten etc. beruht. Das Soziale wird nicht als strittiges Aushandlungsobjekt wahrgenommen, sondern als eigene Sphäre, die durch grundlegende Wahrheiten strukturiert ist. Im Prozess der Konsensfindung wird die Konflikthaftigkeit der Gesellschaft scheinbar aufgelöst (Rancière 2008: 45f.). Dieses Modell setzt Partner_innen voraus, die auf der Grundlage gemeinsam geteilter diskursiver Formen des Austauschs miteinander kommunizieren (ebd.: 36). Kollektive politische Identitäten und der Antagonismus zwischen unterschiedlichen politischen Gruppen werden als Gefährdung einer solchen Gemeinschaft verstanden. Sie werden in der aktuellen politischen Ordnung durch einen breiten Konsens ersetzt und somit vermeintlich überwunden (Mouffe 2005: 5ff.). Postpolitik kann also als Regierungspraxis verstanden werden, die eine Konflikthaftigkeit der Gesellschaft zu negieren versucht, indem durch die Herstellung von Konsens politische Interventionen unterdrückt werden.

Simons und Masschelein diskutieren analog zur ,postpolitischen Kritik' die Bedeutung des Stakeholder in der gegenwärtigen Gesellschaft. Sie identifizieren die Artikulation von Dissens in Form von Interessen als ein zentrales Merkmal der „democracy of stakeholders, with the demos referring to a collective of individuals or interest groups that have a stake or identity in relation to that collective" (2010: 591). Die ,ideale Demokratie', so das allgemeine Verständnis in der Stakeholder-Gesellschaft, sei gegeben, wenn auf Grundlage aller (ausgesprochenen) Interessen, Meinungen und Präferenzen Entscheidungen getroffen werden, die in einem „spirit of consensus“ (ebd.) von allen akzeptiert werden. Übertragen auf den Bereich der Stadtplanung hinterfragt eine solche Kritik die mit dem traditionellen Demokratieverständnis verbundene Notwendigkeit, formelle Partizipationsmöglichkeiten sowie ihr Prinzip der konsensualen Entscheidungsfindung und Integration aller Stakeholder auszubauen (Einig 2005, Mayer 2003). 
Aus Sicht der emanzipatorischen Demokratie sind Partizipationsmöglichkeiten also nicht per se als demokratische Elemente, sondern als Ausdruck der jeweils aktuellen hegemonialen Ordnung zu verstehen. Für eine emanzipatorisch-demokratische Stadtplanung lässt sich daraus die zentrale Forderung ableiten, dass unter Beteiligung nicht nur die Diskussion unterschiedlicher Interessen verstanden wird, sondern dass Raum für die Aushandlung unterschiedlicher Weltbilder geschaffen werden muss. Insbesondere die Frage, auf welcher Grundlage und in welcher Form Entscheidungen über die Gestaltung der Stadt getroffen werden, muss dabei selbst zum Aushandlungsgegenstand werden. Für eine kritische Analyse ist es aus meiner Sicht daher notwendig, Politiken der Partizipation differenzierter zu betrachten. Im Einzelfall ist zu untersuchen, welche Möglichkeiten für das Hinterfragen der bestehenden Ordnung und für die Modifikation und Neuerschaffung von Entscheidungsstrukturen geboten werden. Ebenso wie in der Demokratie stets ein Widerspruch begründet liegt, weil sie niemals fixiert werden kann, ist aus einer solchen Perspektive auch die Partizipation als demokratisches Moment der ständigen Modifikation und Anpassung zu gestalten. Für die politische Praxis bedeutet ein solches Verständnis von Demokratie auch, die Teilnahme an und den Umgang mit Partizipationsangeboten zur Disposition zu stellen.

\section{Das Modell Barcelona}

In Spanien und speziell in Barcelona hat dieZahl der Partizipationsprogramme in der Stadtplanung in den letzten Jahren zugenommen (Bonet i Martí 2012). Barcelona reiht sich damit in eine Liste von Städten ein, die unter der Überschrift von good urban governance [4] Prozesse der Aushandlung zwischen unterschiedlichen Akteur_innen in der Stadtplanung stärken. Im gängigen Narrativ der Geschichte der Stadtplanung Barcelonas werden solche partizipativen Elemente jedoch nicht primär als ein aktuelles Phänomen betrachtet. Vielmehr wird auf die „traditionelle Konsenskultur“ der Stadt verwiesen (Fernández Sánchez 2007: 188), die mit dem Erstarken der Nachbarschaftsbewegungen in den 1970er Jahren begonnen habe. Zentraler Bezugspunkt ist dabei die Umsetzung des ,Plan General Metropolitano' von 1976, der als Beginn einer systematischen Umgestaltung der Stadt nach der Zeit der Diktatur gilt (Monclús 2003: 406). Während der Franco-Ära, die 1975 endete, vollzog sich das Wachstum Barcelonas weitestgehend ohne formelle Stadtplanung. Dadurch herrschte in den 1970er Jahren ein enormer Druck zur Reformierung und Restrukturierung der Innenstadt und der städtischen Peripherie. Die Erstellung und Umsetzung des ,Plan General Metropolitano' wurdevor allem von den Nachbarschaftsvereinen (associació de veïns) forciert, die in vielen Stadtteilen Barcelonas als Reaktion auf die massive Repression während der Diktatur entstanden waren. Mit Unterschriftenaktionen, Demonstrationen und Blockaden forderten sie infrastrukturelle und bauliche Verbesserungen in den einzelnen Vierteln, insbesondere in der Peripherie der Stadt (Kuhn 2012: 47f.). Die Arbeit der Nachbarschaftsbewegungen war von 1970 bis 1979 durch nicht institutionalisierte Formen der Partizipation und Praktiken des Protests geprägt (Bonet i Martí 2012: 16). Während der Diktatur und in den ersten Jahren der Transition zur 
Demokratie hatten die Interventionen der Nachbarschaftsbewegungen in Barcelona nicht nur die Vertretung der Interessen der Bewohner_innen in Bezug auf städtebauliche Maßnahmen im Blick. Sie setzten sich auch dafür ein, dass diejenigen zu Wort kamen, für die das politische System keine Möglichkeit bot, an stadtplanerischen Entscheidungen teilzuhaben (Kuhn 2012: 48). Die Nachbarschaftsbewegungen konnten sich in dieser Zeit als Interessenvertretung der Bewohner_innen etablieren und sich intensiv in die ihr Quartier betreffenden Planungsprozesse einbringen. Diese Rolle als anerkannte Repräsentant_innen der Bevölkerung und Mitwirkende bei der Stadtplanung behielten sie bis in die 1980er Jahre hinein (Cruz i Gallach 2006: 188f.).

Diese Erfahrungen prägten das neue progressive Stadterneuerungsmodell, das mit der ersten demokratischen Regierung 1979 Einzug hielt. Obwohl die Partizipation der Bevölkerung an Stadtplanungsprozessen damals in Barcelona noch keinen institutionellen Rahmen hatte, waren die Anwohner_ innen, vor allem vertreten durch die Nachbarschaftsvereine, de facto als relevante Akteur_innen in die Stadtplanung einbezogen. Über Instrumente wie ,Runde Tische', Konferenzen und Informationsveranstaltungen kam es zu einem regelmäßigen Austausch und einer engen Zusammenarbeit mit der städtischen Verwaltung und den zuständigen Stadtplaner_innen und Architekt_innen (ebd.). Die Ergebnisse dieser Zusammenarbeit, etwa die Ausarbeitung der ,Planes Especiales de Reforma Interior' (PERI), werden als Produkte eines „städtischen Konsens“ (Bonet i Martí 2012: 16) gewertet. Besonderen Ausdruck fand dieser im ,Plan General Metropolitano', der nicht nur als ein Dokument verstanden wird, das im Konsens erzeugt wurde, sondern das auch selbst Konsens erzeugen und unterschiedliche Interessen zusammenbringen konnte (Cruz i Gallach/Martí i Costa 2010: 113). Durch diese Form der konsensualen Stadtplanung wurden viele Forderungen sozialer Bewegungen in stadtpolitische und städtebauliche Maßnahmen umgesetzt (Kuhn 2012: 48). Zusammenfassend wird für den Zeitraum zwischen 1979 und 1983 die praktische Umsetzung von Partizipationsmöglichkeiten für die Bevölkerung und eine zentrale Rolle von sozialen Bewegungen festgestellt (Capel Sáez 2007). Diese Entwicklungen führten zu einem Bild Barcelonas als Stadtgesellschaft mit einer ausgeprägten Konsenskultur, in der Entwicklungsprozesse von der Mehrheit der Bewohner_innen getragen werden (Fernández Sánchez 2007: 188). Bis heute dominiert dieses Bild den vorherrschenden Diskurs und wird noch immer zur Erklärung und Legitimation von Partizipationsprogrammen herangezogen.

Mit der erfolgreichen Bewerbung um die Austragung der Olympischen Spiele (1992) und im Kontext zunehmender Globalisierung und Neoliberalisierung kam es zu einem Wandel in der Stadtentwicklungspolitik (Capel Sáez 2007). Dabei bildete sich ein Leitbild heraus, das insbesondere seit den 1990er Jahren als ,Modell Barcelona' bekannt geworden ist. Während in der Forschung noch diskutiert wird, ob es ein solches Modell überhaupt gibt (Marshall 2000), wird es in der programmatischen Stadtplanung als ein kompaktes und übertragbares Modell gehandelt, das anderen Städten als Vorbild dienen kann (Garcia-Ramon/Albet 2000). Jedoch wird in der Stadtplanungsliteratur darunter nicht immer dasselbe verstanden. Insbesondere variiert die zeitliche Einordnung des 
,Modells Barcelona'. Für lokale Autor_innen, die in vielen Fällen in die Planungsprozesse in Barcelona integriert waren oder weiterhin sind, hatte es seinen Ausgangspunkt bereits in den 1970er Jahren (einige sprechen daher von einer neoliberalen Transition des Modells in den 1980er Jahren). Andere Autor_innen, die das Phänomen eher von außen untersuchen, sehen das Modell als Teil der großen Projekte und neoliberalen Politiken in Zeiten zunehmender Globalisierung und verorten es daher in den 1980er und 1990er Jahren (Monclús 2003: 399).

Das ,Modell Barcelona' gilt als Best Practice und dient vielen Städten als Vorbild dafür, wie es einer bis dato international kaum beachteten Stadt gelingen kann, weltweite Bekanntheit als moderne europäische Metropole zu erlangen (Monclús 2003: 413). Es zeichnet sich vor allem dadurch aus, dass Restrukturierungen der Stadt meist in Form von sogenannten komplexen Projekten (Marshall 2000: 305) organisiert werden. Als komplex werden diese Projekte deshalb bezeichnet, weil sie auf unterschiedlichen räumlichen Planungs- und Wirkungsebenen stattfinden und interdisziplinär durch die Integration von Architektur, Planung und Infrastruktur realisiert werden (ebd.). Großevents wie die Olympischen Spiele 1992 oder das Forum der Kulturen 2004 wurden dabei als strategische Instrumente des Umbaus und der Erneuerung der Stadt genutzt (Monclús 2003). Als zentral bei der Umsetzung der Projekte gilt, dass unterschiedliche regionale Akteur_innen - etwa die Privatwirtschaft, Gewerkschaften oder die Bevölkerung - in die Stadtplanung einbezogen werden (Marshall 2000: 305f.).

Im Kontext dieser neuen Stadtentwicklungspolitik und eingebettet in einen Diskurs der Demokratisierung wurden bestehende Formen der Bürger_innenbeteiligung an der Stadtplanung zunehmend formalisiert und ausgebaut (Bonet i Martí 2012: 17f.). Ausgehend von den nationalen und internationalen Entwicklungen der 1990er Jahre (Rio 1992, Aalborg-Charta) erhielt der Partizipationsbegriff mit den Diskussionen um Nachhaltigkeit eine neue Aufmerksamkeit. Dies schlug sich in Barcelona u. a. in der Einführung der ,Agenda 21' (1998-2002) nieder (ebd.). Zentrale Grundlage für diese Entwicklung sind die,Normas Reguladoras de la Participación Ciudadana'von 1986 (Richtlinien für die Bürger_innenbeteiligung), die,Ley de Participación Ciudadana' (das Gesetz zur Partizipation der Bewohner_innen der Stadt), das seit 2002 einen konkreten legislativen Rahmen für die Partizipation der Bevölkerung bietet, und der ,Plan Director Municipal de Participación Ciudadana' von 2010 (der Gemeindeentwicklungsplan für die Bürger_innenbeteiligung), der Vorschläge und Richtlinien für die Verwaltung umfasst. In diesen Dokumenten werden die formalen Partizipationsmöglichkeiten als wichtige Elemente einer demokratischen Stadt dargestellt. So heißt es in der Einleitung zum ,Plan Director Municipal de Participación Ciudadana':

„In the city of Barcelona, there is currently a broad consensus among all municipal political groups, people and associations with representation on the various municipal councils on the need for a more ambitious approach that acknowledges citizen participation as a key aspect of democracy.“ (Ajuntament de Barcelona 2010: 6)

In öffentlichen Publikationen der Stadt sowie in großen Teilen der Fachliteratur werden solche Formen formaler Partizipation als 
Fortführung der konsensualen Stadtpolitik der 1970er und 1980er Jahre dargestellt. Durch sie soll eine partizipative Politik garantiert und auf Dauer gestellt werden. Die Geschichte der Stadtentwicklungspolitik wird dabei meist als kontinuierlicher Prozess erzählt, in dem die Bewohner_ innen seit der Zeit der Transition zunehmend in die Stadtplanung einbezogen werden. Der Bezug auf die Tradition der Bürger_innenbeteiligung in Barcelona und die Verankerung in den Jahren der demokratischen Transition in Spanien legitimieren die aktuelle Stadtpolitik und betten sie in einen Diskurs der Demokratisierung ein. Betrachtet man jedoch die tatsächlichen Beteiligungsmöglichkeiten der Bevölkerung, zeigt sich ein anderes Bild: Sie nahmen seit den 1990er Jahren ab (Bonet i Martí 2012, Cruz i Gallach/Martí i Costa 2010, Marshall 1996). Dabei wird aus Sicht einer partizipativen Demokratie außerdem ein Wandel des Partizipationsbegriffs seit den 1970er Jahren deutlich. So scheinen die Interventionen der Bevölkerung - in vielen Fällen Aktivitäten im Rahmen der Nachbarschaftsvereine - in den letzten Jahren der Diktatur und zur Zeit der Transition zur Demokratie durchaus transformativen Charakter gehabt zu haben. Über die Nachbarschaftsvereine beteiligte sich die Bevölkerung in den 1970er und 1980er Jahren noch stark an der Gestaltung und Entwicklung der Stadt. Es gelang der Bevölkerung dabei, gleichzeitig ihre Rolle in der Stadtplanung zu definieren und zu verteidigen. Die Bewohner_innen prägten das Stadtentwicklungsmodell dieser Zeit und stellten sich - als Nachbar_innen (vecinas/vecinos) - selbst als politische Subjekte dar.

Die Proteste der Nachbarschaftsvereine in den 1970er Jahren sind als Ursprung der heutigen Partizipationskultur positiv konnotiert in die Geschichte der Stadtentwicklung Barcelonas aufgenommen worden. Spätere Versuche der Bewohner_innen, nicht nur über offizielle Partizipationsmöglichkeiten, sondern auch mit anderen Mitteln auf die Stadtplanung Einfluss zu nehmen und ihre Wünsche und Vorstellungen von der Gestaltung der Stadt einzubringen, bleiben in dieser Erzählung dagegen außen vor. Dabei waren fast alle städtebaulichen Maßnahmen in Barcelona - von den großen Stadtumbauten im Rahmen der Olympischen Spiele 1992 und des Forums der Kulturen 2004 über kleinere Projekte der Stadterneuerung bis hin zu aktuellen Entwicklungsprozessen - von teilweise massiven Protesten begleitet. Die Nachbarschaftsvereine, also die traditionell wichtigsten Akteur_innen in diesen Protesten, erlebten in den 1990er Jahren (vgl. Acebal 2008) sowie seit $2011 \mathrm{im}$ Zuge der Dezentralisierung der 15-M-Bewegung Phasen des Wiedererstarkens. Eine weitere relevante Akteurin ist die Hausbesetzer_innenbewegung. Ende der 1990er und zu Beginn der 200oer Jahre entstanden in Barcelona eine Reihe neuer Protestplattformen, die sich aus Vertreter_innen von alten und neuen sozialen Bewegungen zusammensetzen, teilweise ergänzt - infolge eines lokalen Aufwertungsprozesses in den letzten Jahren - durch neu hinzugezogene gentrifier (Pascual-Molinas/Ribera-Fumaz 2009: 185). Diese Protestplattformen bilden große Netzwerke, die in vielen Fällen auch mit sympathisierenden Spezialist_innen zusammenarbeiten, welche mit Gutachten und wissenschaftlichen Untersuchungen die Argumente der Protestbewegungen stützen sollen (Cruz i Gallach 2006: 187f.). 


\section{Die Marina Port Vell}

Einer der Orte in der Stadt, an denen aktuell Protest deutlich wird, ist das innerstädtische Hafengebiet Port Vell. Port Vell ist die heutige Bezeichnung für das Gebiet, auf dem ab dem 15. Jahrhundert Barcelonas erster Handelshafen entstand. Dieser Teil des Hafens, der ab den 1980er Jahren als ,Bürgerhafen“ der Bevölkerung zugänglich gemacht wurde und seitdem als Sporthafen für kleine und mittelgroße Segelboote genutzt wird, soll nach den Plänen der Hafenverwaltung zukünftig zu „one of the leading superyachts destinations in the world“ (Marina Port Vell 2011) ausgebaut werden und so „the highest standard of service and security for our guests and their yachts" (ebd.) garantieren. Neben dem Bau einer im Wasser liegenden, nicht öffentlich zugänglichen Ebene für Parkplätze und Versorgungseinrichtungen sind auch zwei neue Gebäude geplant. Die Umbaumaßnahmen, die von den Konzessionären von Port Vell, Salamanca Group, in enger Zusammenarbeit mit der Hafenverwaltung und Mitgliedern der Stadtverwaltung geplant wurden und durchgeführt werden, haben bereits begonnen und sollen 2014 abgeschlossen werden.[5]

Als Informationen über den geplanten Umbau der Marina Port Vell bekannt wurden, gründete sich auf Initiative eines Nachbarschaftsvereins des Stadtviertels La Barceloneta und Aktivist_innen der Hausbesetzer_innenbewegung im Jahre 2012 die Protestplattform ,Plataforma Defensem el Port Vell' (Plataforma). Die Plataforma versammelt als zentrale Akteurin in den Protesten unterschiedliche Kritiken am Projekt. Im Jahre 2013 wurde sie weitgehend durch die neu gegründete Protestgruppe ,Asamblea Portuaria Vecinal' (APV, Versammlung der Nachbar_innen des Hafens) abgelöst. Beide Gruppen organisieren zivilgesellschaftlichen Widerstand gegen das Bauprojekt, nehmen aber auch an partizipativen Verfahren teil, zu denen vonseiten der städtischen Behörden eingeladen wird. Zentrale Kritikpunkte sind die mit den Umbaumaßnahmen verbundenen Prozesse der Privatisierung und Kommodifizierung öffentlichen Raums, die Errichtung einer abgezäunten und durch privates Sicherheitspersonal gesicherten Zone und die drohende exklusive Nutzung dieser durch ,Superreiche'. All diese Aspekte werden als Teil eines neuen Stadtentwicklungsmodells gesehen, das als ,Ausverkauf der Stadt für den Tourismus' beschrieben wird. Die Neugestaltung von Marina Port Vell, so die Gegner_innen, „macht wieder einmal die Beharrlichkeit deutlich, mit der ein neues Stadtmodell gefördert wird, in welchem Stadt exklusiv und privatisiert und nur für einige ,Superreiche“ zugänglich ist“ (Plataforma Defensem el Port Vell 2012; Übers. d. A.).

Die Umgestaltung des Yachthafens steht für viele stellvertretend für die Umsetzung dieses Modells. Als weitere Beispiele wird auf andere bereits umgesetzte Großprojekte im Hafen verwiesen, wie das Hotel Vela, das Einkaufszentrum Maremagnum oder die Filiale des Modelabels Desigual. Auch der neue Flächennutzungsplan der Altstadt oder die Pläne für eine Umgestaltung der Avenue Paral-lel werden als aktuelle Beispiele für ein solches Stadtmodell genannt. Die Gegner_innen des Umbauprojekts lehnen dessen Zielsetzung insbesondere mit Bezug auf die erwarteten „sozialen Auswirkungen, nicht nur im Stadtviertel, sondern in der ganzen Stadt" ab (Plataforma Defensem el Port Vell 2012; Übers. d. A.). Zu den befürchteten 
Folgen zählen die Verdrängung der (ursprünglichen) Bevölkerung aus den Innenstadtvierteln und der Rückgang der traditionellen Fischerei. Letzteres, so warnen die Protestierenden, werde sowohl die Ernährungssouveränität als auch die maritime Kultur der Stadt, die als wichtige Bestandteile des kulturellen und sozialen Erbes Barcelonas gesehen werden, nachteilig beeinflussen.

Ähnlich wie in anderen aktuellen städtebaulichen Konflikten geht die Kritik am Projekt jedoch insofern über die reine Interessensartikulation hinaus, dass die Protestierenden ihr eigenes Recht (und das aller Bewohner_ innen der Stadt) einfordern, über alle Entscheidungen bezüglich der Stadtentwicklung informiert und in die Entscheidungsfindungsprozesse der Stadtplanung einbezogen zu werden. Eine Gegnerin des Projekts betont: „Noch vor jeder anderen Kritik haben wir kritisiert, nicht einbezogen worden zu sein. Wir lehnen jeden Plan ab, egal wie er aussieht, wenn wir nicht in die Planung einbezogen werden." [6] Dieses Recht mitzubestimmen wird einerseits von den Konzepten des ,Rechts auf Stadt' abgeleitet, andererseits von einer spezifischen lokalen Vorstellung von Nachbarschaft, die etwa in den Begriffen des barrio (Stadtviertel) und der vecinos/vecinas (Nachbar_innen) zum Ausdruck kommt.

Indem sie sich auf ihr ,Recht auf Stadt' (nuestro derecho a la ciudad) beziehen, verweisen die Aktivist_innen zum einen auf die international in vielen Städten in den letzten Jahren aufgekommenen Bewegungen, die sich unter diesem Schlagwort formiert haben. Zum anderen erheben sie Anspruch auf eine konflikthafte Aushandlung darüber, wie die Stadt gestaltet werden soll. Ein weiterer Aspekt, den die Gegner_innen des Projekts mit Bezug auf ihr ,Recht auf Stadt' betonen, ist der Anspruch auf öffentliche Räume, in denen Freizeit selbst gestaltet und ohne den Zwang zu konsumieren verbracht werden kann. Die spezifische Bedeutung, die der Nachbarschaft in den barrios zugeschrieben wird, kann auf die lange Tradition der Nachbarschaftsbewegungen zurückgeführt werden, denen (wie im zweiten Abschnitt dieses Beitrags beschrieben) eine wichtige Rolle bei den Protesten um Stadtentwicklungsprojekte zukommt.

Wichtig ist auch die Vorstellung von maritimidad, die auf das Konzept der maritimité zurückgeführt wird.[7] Dieses wiederum bezieht sich auf die aktuelle und historische Beziehung zwischen den Alltagspraktiken der Bewohner_innen einer Stadt und dem Meer. Im Protest wird dieser Begriff aufgenommen, steht hier jedoch für eine allgemeinere Vorstellung von einer maritimen Kultur:

„Die Stadt Barcelona war immer schon grundlegend mit dem Meer verbunden. Es ist eine Hafenstadt, eine Küstenstadt. [...] Es geht also darum, dieses kulturelle Erbe aller zurückzugewinnen. Es geht um einen elementaren Bestandteil der Stadt, der als Teil des kollektiven Gedächtnisses der Bewohner_innen mit Barcelona verbunden ist. [...] [Port Vell] ist einer der wenigen Räume, die uns von dem, was maritimidad der Stadt war, geblieben sind, und welche jetzt ebenfalls verlorengehen. Eine unserer Forderungen ist daher das Thema der maritimidad als bestimmendes Merkmal der Stadt an sich. [...] Es ist ein Recht auf ein kulturelles Erbe - oder sagen wir: ein historisches Kulturerbe.“ [8] 
Verzahnt mit diesen Konzepten beschreiben die Gegner_innen der Umbaupläne ihr eigenes politisches Projekt als ,echte Demokratie mit einer ,echten Partizipation'. Bezogen auf Port Vell wird dabei das Ziel verfolgt „Port Vell als echten Stadt- und Bürgerhafen zurückzugewinnen, indem wir die demokratische Kontrolle über ihn erlangen. [...] [Wir möchten] diese Realität von unten nach oben errichten." [9] Der Begriff der ,echten Demokratie' verweist auf die Massenproteste, die unter dem Namen Indignados- oder 15-M-Bewegung ab 2011 bekannt geworden sind. Entsprechend wird von den Gegner_innen des Bauprojekts direkt auf diese Bewegung Bezug genommen: „Was wir wollen ist zu demokratisieren, echt zu demokratisieren: die Stadt, die Politik - und das ist eine der Forderungen, die vom 15-M kommt, nicht wahr? iDemocracia real ya! [Echte Demokratie jetzt!].“ [10]

Zentral ist bei diesem Konzept die Idee einer Entscheidungsstruktur, in der alle Bewohner_innen die Möglichkeit haben, ihre Vorstellungen davon, wie die Stadt sich entwickeln soll, jederzeit einzubringen:

„Echte Partizipation bedeutet, dass [...] vom ersten Keim an, der ersten Idee, dass in einem Viertel etwas getan werden muss - ohne dass es bereits ein Projekt gibt, ohne jegliche vorausgehende Diagnose - die Vereine des Viertels darüber informiert werden, dass darüber nachgedacht wird, möglicherweise eine Reform durchzuführen. Und dass sie sagen, lasst uns darüber sprechen, wir fangen mit euch an mal schauen, ob wir es machen oder nicht. Und wir können an einer möglichen Reform, einer möglichen Veränderungen teilhaben. Das ist echte Partizipation für uns.“ [11]

Diese ,echte Partizipation' wird nicht nur von staatlichen Stellen eingefordert, sondern sie wird auch in alternativen Praktiken umgesetzt, wie beispielsweise in öffentlichen Zukunftsworkshops oder horizontal strukturierten asambleas (Versammlungen). Zentral ist für die Anwohner_ innen dabei, darauf aufmerksam zu machen, dass es alternative Formen der Entscheidungsfindung gibt und dass die aktuellen Macht- und Entscheidungsstrukturen nicht die einzig möglichen sind:

„Wir [die Plataforma Defensem el Port Vell] versuchen, keine Führungspersonen zu haben, das ist wichtig. Sagen wir, eine Besessenheit von [der Idee] der Horizontalität. Aber wieso? Weil diese Form der Demokratie, die es bis heute gegeben hat, dir deine Repräsentativität genommen hat. Sie sagen dir: Du muss alle vier Jahre wählen - und das war's. [...] Wir müssen einen reset machen, eine Volksversammlung gründen und schauen, wie es dann weitergeht. Wenn dein Computer nicht mehr funktioniert, musst du einen Neustart machen. [...] Das hier hat nicht geklappt, also machen wir einen Neustart und fangen von vorne an.“ [12]

Dieses gegenhegemoniale politische Projekt wird im Verhältnis zu und gleichzeitig in Abgrenzung von der aktuellen politischen Ordnung entwickelt, die als eine Ordnung charakterisiert wird, die sich zwar selbst als partizipativ und demokratisch bezeichnet, sich jedoch in Wirklichkeit als korrupte und undemokratische Plutokratie darstellt. Bestehende 
Partizipationsprogramme in der Stadtplanung werden hingegen als Legitimationsstrategie der Stadtregierung identifiziert. Eine Teilnahme wird daher (zumindest teilweise) abgelehnt. Die Gegner_innen des Bauprojekts wenden sich nicht nur gegen die vorherrschenden politischen Entscheidungsprozesse, sondern auch dagegen, dass diese als alternativlos dargestellt werden: „Sie präsentieren uns das Projekt der großen Yachten, als gäbe es keine Alternative. Als wäre es dieses oder keines“.[13]

Verbunden mit dem Ideal einer ,echten Partizipation“ ist auch die Forderung, die geographischen Zuständigkeiten und Verwaltungsbereiche im Hafengebiet zu restrukturieren. Der Yachthafen Port Vell steht nicht unter städtischer Verwaltung, sondern untersteht als Teil des Hafengebietes der Entscheidungsbefugnis der staatlichen Hafenverwaltung. Folge der besonderen verwaltungstechnischen Zuteilung ist, dass Entscheidungen bezüglich des Port Vell nur dann den formellen Vorgaben für die Partizipation der Stadtbewohner_innen unterliegen, wenn sie von der Stadtregierung bestätigt werden müssen.

Ein großer Teil der Entscheidungen, welche die Reformen und den Umbau des Port Vell betreffen, werden von der Hafenverwaltung getroffen. Die Entscheidungsstrukturen der Hafenverwaltung, einer komplexen Organisation, die teilweise als Unternehmen ,Port 2000“ fungiert, in dem aber auch Vertreter_innen der Stadtregierung sitzen, werden von den Gegner_innen als undurchsichtig und undemokratisch beschrieben, ohne eine Möglichkeit der Bevölkerung, an Entscheidungen teilzuhaben. So führt die Verwaltungsstruktur dazu, dass der Hafen als ,antidemokratischer Raum' wahrgenommen wird:

„Der Paseo de Colon [die Straße, die Hafen und Stadt trennt] ist eine antidemokratische Trennlinie. [...] Dahinter beginnt ein antidemokratischer Raum - ein Raum, in dem die Bürger_innen der Stadt Barcelona nicht handeln können, selbst wenn die Aktivitäten am Hafen uns stark betreffen“.[14]

Die Projektgegner_innen fordern deshalb die Stadtregierung auf, die Verwaltung und Entscheidungskontrolle über den Port Vell zu übernehmen und diesen Teil des Hafens in den städtischen Raum zu integrieren. Dadurch, so die Vorstellung, könne dieser Bereich demokratischer gestaltet werden.

Während Helena Cruz i Gallach (2006: 192) in ihrer Analyse von urbanen Protesten in Barcelona einen Trend zu zwei zeitlich aufeinanderfolgenden Phasen identifiziert - eine erste konfrontative Phase, in der Stadtplaner_innen, Initiator_innen und Investor_innen mit den von den Plänen abweichenden Interessen der Protestierenden konfrontiert werden, und eine zweite kooperative Phase, in der die Akteur_innen aufeinander zugehen und beginnen, miteinander zu verhandeln -, zeigt sich im Fall Port Vell ein komplexes Zusammenspiel von Partizipation ,von unten“ und Partizipation ,von oben'. Auf der einen Seite suchen die Protestierenden den Kontakt und die Kommunikation mit den städtischen Behörden und nutzen dabei formelle Beteiligungsinstrumente und -kanäle, die von der Stadtregierung auf Basis der rechtlichen Grundlagen eingerichtet worden sind. Auf der anderen Seite entzündete sich Kritik an den Bedingungen der formellen Partizipation. Ein Kritikpunkt betrifft 
die finanziellen Hürden für die Bürger_innenbeteiligung: Die genauen Pläne für die Umbaumaßnahmen waren nicht frei zugänglich, sondern mussten käuflich erworben werden. Erst nach starken Protesten wurden sie gegen einen ,symbolischen Betrag' zur Verfügung gestellt. Weitere juristische Schritte gegen die Umbaupläne scheiterten zudem, berichtete ein die Aktivist_innen beratender Anwalt im Interview, an den hohen Gebühren, die für die Einreichung von Klagen gezahlt werden müssen. Die Gegner_innen des Projekts entschieden deshalb in einigen Fällen, sich an den partizipativen Verfahren nicht zu beteiligen.

$\mathrm{Zu}$ den partizipativen Verfahren im Fall Port Vell gehören mehrere Gespräche zwischen Anwohner_innen und Hafenverwaltung/ Stadtverwaltung, eine Begleitkommission des Bauprojekts (,Comisión de Seguimiento') unter Beteiligung der Nachbarschaftsvereine, der Hafenverwaltung und der Stadtverwaltung sowie die öffentliche Ausstellung der Umbaupläne für diesen Hafenbereich und die Möglichkeit, dazu Änderungsvorschläge einzubringen. Letzteres bezieht sich aufgrund der Entscheidungsstruktur im Hafengebiet nur auf den Sondernutzungsplan, ohne den ein Teil der im Zuge der Umbauten geplanten Gebäude nicht errichtet werden kann und der vom Stadtrat bestätigt werden muss. In diesen unterschiedlichen Verfahren wurden die Interessen verschiedener Akteursgruppen diskutiert, wie der Wunsch der Anwohner_innen und der anliegenden Restaurants, dass die Sicht auf den Hafen und den Horizont nicht durch direkt am Ufer ankernde Yachten versperrt wird oder die Forderung der Bewohner_innen der angrenzenden Stadtviertel nach Arbeitsplätzen. Es wurde versucht, Lösungen zu finden, die für alle Beteiligten annehmbar sind. So wurde beispielsweise beschlossen, im Viertel La Barceloneta Bildungseinrichtungen zu schaffen, in denen die Jugendlichen für die Arbeit im neuen Yachthafen qualifiziert werden. Auch wurden Regelungen für die Anordnung der Boote im Hafen getroffen, mit denen die Auswirkungen auf die Aussicht der Anwohner_innen reduziert werden. Initiativen, die das Entwicklungsmodell der Stadt und das Projekt Port Vell komplett ablehnen, fanden jedoch kein Gehör. Deren Position wurde lediglich als mangelnde Bereitschaft zum Dialog über den Konflikt wahrgenommen, wie das folgende Zitat beispielhaft zeigt:

„Sie verhalten sich kämpferisch, gegen das Projekt Port Vell. Und da sie dagegen kämpfen, können wir sagen, es gibt keinen Dialog. Sie kommen zu den Versammlungen der Kommission, um zu protestieren. [...] Sie kommen nicht, um irgendetwas zu unterstützen oder um zuzuhören, was man sagt. [...] Mitten in der Versammlung stehen sie auf und gehen. [...] Aber es sind nicht alle Nachbarn gegangen, nicht im Geringsten. Die von den Nachbarschaftsvereinen der Gegend sind geblieben [...]. Und diese sind in einen Dialog mit uns getreten.“ [15]

Auf ihrer Homepage bezeichnet das Stadtparlament Barcelonas die im Februar 2012 ins Leben gerufene ,Comisión de Seguimiento' als partizipativen Prozess (proceso de participación), in den Interviews wird das Verfahren allerdings sowohl von den daran teilnehmenden Gegner_innen des Projekts als auch von der städtischen Verwaltung selbst als reine Informationsveranstaltung beschrieben, auf der die Anwohner_innen über 
den Verlauf der Bauarbeiten informiert werden. Die Projektgegner_innen forderten von Anfang an, dass bereits vor der Genehmigung des Projekts im Stadtparlament ein Beteiligungsverfahren durchgeführt wird. Die Ablehnung dieser Forderung wurde von den Mitgliedern der Stadtregierung im Interview damit begründet, dass die Stadt im Fall Port Vell ein konkretes Ziel (nämlich wirtschaftliches Wachstum) verfolge und bereits konkrete Pläne habe. Partizipationsprogramme hingegen hätten die Funktion, der Stadt bei einer Entscheidung zu helfen, wenn diese noch keine Pläne habe, und seien deshalb für den Fall Port Vell ungeeignet.

Zusammenfassend sind unterschiedliche Faktoren zu benennen, die die ,wirkliche' Beteiligung der Bevölkerung einschränken: Erstens bestehen finanzielle Hürden (z. B. Kosten für den Erwerb von Informationen). Zweitens ist die Form, in der sich die Bevölkerung einbringen kann, stark vorgegeben. Mit zunehmender Institutionalisierung und Formalisierung wird die Rolle der Anwohner_innen als Mitwirkende an der Stadtplanung auf die Teilnahme an bestimmten vorgegebenen Prozessen verengt. Dabei wird von der Stadtverwaltung bestimmt, wer zu den jeweiligen Verfahren und Versammlungen eingeladen wird (beispielsweise welche Organisation die Anwohner_innen vertreten soll und ob einzelne Individuen zugelassen sind), welche Entscheidungen getroffen werden und teilweise sogar zwischen welchen Entscheidungsmöglichkeiten gewählt werden kann. Unterschiedlichen Phasen eines Bauprojekts werden dabei unterschiedliche Entscheidungen zugeordnet (in der ,Comisión de Seguimiento' wird beispielsweise nicht mehr diskutiert, ob das Bauprojekt realisiert wird.). Raum, die Macht- und Entscheidungsstrukturen zu reflektieren und zu hinterfragen, besteht nicht. Drittens ist Partizipation nur in einem beschränkten Rahmen vorgesehen, bestimmte Grundlagen werden vorausgesetzt. Ähnlich wie in Barcelona eine ,demokratische Stadtpolitik (im traditionellen Verständnis von Demokratie) als alternativlos angesehen wird, ist es auch unhinterfragtes Ziel, dass die städtischen Politiken das Wirtschaftswachstum der Stadt fördern sollen (Marshall 1996). Viertens ist auch die Form der Artikulationsmöglichkeiten in den verschiedenen Partizipationsverfahren vorgegeben. Indem die Partizipationsprogramme eine Integration (der Interessen) aller Stakeholder versprechen, legen sie als einzig mögliche Form, Dissens zu artikulieren, die Aushandlung zwischen unterschiedlichen Interessensvertreter_innen fest.

Im Fall von Port Vell wurden durch unterschiedliche Prozesse der Bürger_ innenbeteiligung Möglichkeiten gefunden, verschiedene Interessen ,miteinander zu versöhnen', so dass der Wunsch nach geringer Beeinträchtigung des Sichtfelds durch die baulichen Veränderungen und die Forderungen nach mehr Arbeitsplätzen den Umbaumaßnahmen nicht länger im Wege standen. Im Partizipationsprozess wurden also keine Räume geschaffen, in denen unterschiedliche hegemoniale Projekte miteinander konkurrierten, sondern solche, in denen Interessen artikuliert und Interessenskonflikte aufgelöst wurden, indem vermeintlich für alle die besten Lösungen gesucht und gefunden wurden. Es fand dabei eine Aufteilung der Welt auf der Grundlage von Interessen als spezifische Form der Konstituierung der Gesellschaft statt. So konnten scheinbar alle ihre Vorstellungen einbringen und im Konsens waren grundlegende Entscheidungen und Veränderungen möglich. Tatsächlich 
waren jedoch bereits vor dem Beginn der Aushandlungsprozesse gewisse Grenzen festgelegt. Das Beispiel zeigt, wie die polizeiliche Ordnung (im Rancière'schen Sinne) bestimmt, wer an der Gestaltung der Stadt Teil hat, und diesen Personen einen bestimmten Rahmen bietet, in dem sie sich Gehör verschaffen können - aber auch gleichzeitig diejenigen stimmlos macht, die nicht gehört werden (sollen). Durch die vermeintliche Integration aller, wird überdeckt, dass es Personen und Ideen gibt, die aus den Entscheidungsprozessen ausgeschlossen sind.

Die partizipativen Verfahren bieten in diesem Fall keine Möglichkeit für eine radikale Kritik - also Interventionen, die ,außerhalb des Rahmens 'liegen - denn da es kein Außen gibt, wird jeder Konflikt als Interessenskonflikt markiert, in das polizeiliche System integriert und vermeintlich gelöst. Indem jeder Dissens in der Gesellschaft als Interessenskonflikt verstanden wird, bleibt als einzige Form des Sprechens die Artikulation von Interessen, Meinungen und Präferenzen. Im Kontext dieser spezifischen polizeilichen Ordnung werden die einzelnen Individuen zu Subjekten, die ihre Interessen vertreten sehen wollen - zu Stakeholdern - und bestätigen gleichzeitig im Subjektivierungsprozess die herrschende Ordnung. Demokratische Interventionen, die eine Gefahr für die polizeiliche Ordnung darstellen, werden auf diese Weise gebändigt. Partizipationsprogramme können daher als Teil der gouvernementalen Systemstabilisierungspolitik der Polizei verstanden werden. Gleichzeitig sind die partizipativen Verfahren auch ein Instrument der Regierenden, mit dem auf das Wissen der Anwohner_innen über die sie umgebende Umwelt zugegriffen werden kann. Mit diesem Wissen kann eine bessere Stadtplanung gemacht werden, denn

„der Planer, der in seinem Büro eingeschlossen ist, hat nicht das Wissen der Bewohner_innen. [...] Wer dort [an einem Platz, der umgestaltet werden soll] lebt, sind die Nachbarn dieses Platzes. Sie kennen die Bedürfnisse und wissen, was auf diesem Platz passiert.“ [16]

Auf dieses Wissen soll nach den Angaben von Stadtregierung und -verwaltung jedoch nur dann zurückgegriffen werden, wenn die Stadt noch nicht „ein Projekt a priori“ [17] hat und noch keine bestimmten Ziele verfolgt.

Die Protestierenden zeigen dagegen, dass unterschiedliche Vorstellungen über Stadtentwicklung bestehen: Es manifestiert sich Dissens. Dabei zeigen sich nicht nur unterschiedliche Interessen bezüglich der konkreten Ausgestaltung der Stadtentwicklung, sondern auch verschiedene Vorstellungen darüber, wer bestimmt, wie Stadt gestaltet sein soll, und wer nicht, also wie die aktuell bestehende Ordnung der Stadtentwicklung strukturiert ist. Die Aktivist_innen setzen sich massiv dafür ein, in den Entscheidungen über die Entwicklung der Stadt mitzubestimmen. Mit den Worten Rancières lässt sich sagen: Diejenigen, die nicht sprechen können, erheben die Stimme. Auch wenn nicht jede Partizipation ,von unten' in Barcelona einen transformativen Charakter aufweist (Bonet i Martí 2012: 23), zielen doch zumindest im dargestellten Beispiel die Proteste darauf ab, Macht- und Entscheidungsstrukturen zu verändern und die Städter_innen selbst als politische Akteur_innen, die ihre Stimmen erheben, zu positionieren. Dabei wird deutlich, dass Proteste das Potenzial haben können, 
den ,Rahmen des Möglichen` infrage zu stellen und zur Politisierung von Stadtentwicklung beizutragen.

\section{Fazit}

Mein Beitrag zeigt am Beispiel Barcelona und dem Port Vell, wie scheinbar inkludierende Stadtpolitiken Ausschlüsse produzieren. Partizipation ist möglich - allerdings nur innerhalb eines vorgegebenen Rahmens. Forderungen nach einer Veränderung der aktuellen politischen Ordnung, der Anspruch auf eine ,echte Partizipation' werden nur noch als fehlende Bereitschaft zum Dialog wahrgenommen - sie werden zum ,Lärm‘ im Sinne Rancières. Vor diesem Hintergrund muss das hegemoniale Narrativ der Stadtentwicklung Barcelonas, das als eine kontinuierliche Weiterentwicklung und Umsetzung von Beteiligungsmöglichkeiten für die Bevölkerung erzählt wird, revidiert werden.

Eine emanzipatorisch-demokratische Perspektive macht qualitative Unterschiede zwischen verschiedenen historischen Phasen und Formen von Partizipation deutlich. In den 1970er und 1980er Jahren scheint die Bevölkerung in politischen Momenten auf die Gestaltung der Stadtentwicklung und die bestehenden Macht- und Herrschaftsstrukturen eingewirkt und so die hegemoniale Ordnung in gewisser Weise mitgeprägt zu haben. Indem sie das Wort ergriffen und öffentlich Anspruch darauf erhoben, mit ihren Wünschen und Bedürfnissen gehört zu werden, konstituierten sich die Bewohner_innen der Stadt unter dem Begriff der/des vecinos/vecinas als politisches Subjekt. Demgegenüber zielen die heutigen, stark formalisierten Partizipationsmöglichkeiten auf die Herstellung eines rationalen Konsenses. Forderungen nach einer Änderung der Entscheidungsstrukturen, die über die Interessensartikulation in Bezug auf ein spezifisches Bauprojekt hinausgehen und daher von einem solchen Konsens nicht, eingefangen' werden können, finden keinen Eingang in diese Prozesse. Das Beispiel Port Vell zeigt, dass erst im Protest - der Partizipation ,von unten“ - solche Forderungen möglich werden. In der Aktion selbst, in der der Widerstand gegen das Bauprojekt und gegen die dahinterstehenden Entscheidungsstrukturen öffentlich gemacht wird, bietet sich die Chance, der postpolitischen Ordnung ,echte Demokratie‘ als gegenhegemoniales Projekt entgegenzusetzen. Die offizielle Geschichtsschreibung der Stadtentwicklung Barcelonas, die sich mit dem Hinweis auf die partizipativen Möglichkeiten zur Zeit der demokratischen Transition legitimiert, geht auf solche Unterschiede nicht ein.

\section{Endnoten}

[1] Der Beitrag greift auf eigenes empirisches Material zum Fall Marina Port Vell zurück, das bei zwei Forschungsaufenthalten in Barcelona 2012 und 2013 erhoben wurde. In dieser Zeit wurden Leitfadeninterviews u. a. mit Vertreter_innen der Stadtregierung und Abgeordneten der im Stadtrat vertretenen Parteien, Mitarbeiter_innen der Stadtund der Hafenverwaltung, aktiven Anwohner_innen und Mitgliedern unterschiedlicher Protestgruppen durchgeführt, ergänzt durch teilnehmende Beobachtungen bei mehreren Protestgruppen und -veranstaltungen. Zur Auswertung kamen auch weitere relevante Dokumente wie Zeitungsartikel, Internetbeiträge, parlamentarische 
Unterlagen und solche der Stadtverwaltung sowie wissenschaftliche Publikationen zum Fall Port Vell. Der Schwerpunkt der Analyse liegt auf der Rekonstruktion des Protests und seinen Veränderungen im Zeitverlauf, auf den zentralen Akteur_innen, Diskursen und Praktiken sowie auf deren Wechselbeziehungen.

[2] Bonet i Martí macht darauf aufmerksam, dass beide Formen in vielfältiger Weise miteinander verschränkt sein können.

[3] Im Folgenden wird in diesem Beitrag die Bezeichnung Postpolitik verwendet. Auf das Problem der uneinheitlichen Verwendung der Begriffe und die Frage, ob die Bezeichnungen für das Gemeinte überhaupt sinnvoll sind, gehen Schipper und Mullis in dieser Ausgabe ein. Zum Verständnis des Präfixes ,post‘ vgl. Meyer 2011: 22.

[4] Für einen Überblick zum Begriff urban governance vgl. Schnur/Drilling 2009.

[5] Diese Informationen wurden im Interview mit einer Vertreterin der Salamanca Group sowie mit Vertreter_innen der Hafenverwaltung bestätigt.

[6] Auszug aus einem Beobachtungsprotokoll vom 13.6.2013.

[7] Interview mit J. M., Mitglied der Plataforma.

[8] Interview mit M. F., Bewohnerin des Stadtviertels La Barceloneta, Mitglied der Plataforma und der APV (Übers. d. A.).

[9] Vortrag der APV auf der Diskussionsveranstaltung ,Seminari Geocrítica' am 18.6.2013 (Übers. d. A.).

[10] Interview mit M. E., Mitglied der Plataforma und der APV (Übers. d. A.).

[11] Interview mit P. P., Bewohnerin des Stadtviertels La Barceloneta und Mitglied der Plataforma, der APV und eines Nachbarschaftsvereins (Übers. d. A.).

[12] Interview mit M. E. (Übers. d. A.).

[13] Francesc Magrinyà, zit. nach Pauné 2013 (Übers. d. A.).

[14] Interview mit M. E. (Übers. d. A.).

[15] Interview mit Carles Agustí, Leiter der Kommission für Bürgerbeteiligung und Verbände (Comissionat de Participació Ciutadana i Associacionisme) der Stadt Barcelona (Übers. d. A.).

[16] Ebd.

[17] Ebd.

\section{Autor innen}

Catarina Gomes de Matos ist Soziologin und Stadtgeografin und beschäftigt sich mit urbanen Konflikten und Protesten. Sie ist wissenschaftliche Mitarbeiterin am Institut für Umweltsozialwissenschaften und Geographie in Freiburg.

catarina.matos@geographie.uni-freiburg.de

Dank gilt der Baden-Württemberg Stiftung für die finanzielle Unterstützung der Forschungsarbeit im Rahmen des Programms für Post-Doktorand_innen und Post-Doktoranden (Projektleitung: Samuel Mössner) und dem DAAD für seine Förderung des Auslandsaufenthalts.

\section{Literatur}

Acebal, Marc Andreu (2008): Moviments socials i crítica al ,Model Barcelona'. De l'esperança democràtica de 1979 al miratge olímpic de 1992 i la impostura cultural del 2004. In: Scripta Nova. Revista electrónica de Geografía y Ciencias Sociales XII 270(119).

Ajuntament de Barcelona (2010): Municipal Master Plan for Citizen Participation 2010-2015. www.bcn.cat/participacio/pdf/2011PDMPCangles11.03.11.pdf (letzter Zugriff am 27.4.2013)

Alt, Christian/Teubner, Markus/Winklhofer, Ursula (2005): Partizipation in Familie und Schule - Übungsfeld der Demokratie. In: Aus Politik und Zeitgeschichte 41, 24-34. 
Bonet i Martí, Jordi (2012): El territorio como espacio de radicalización democrática. Una aproximación crítica a los procesos de participación ciudadana en las políticas urbanas de Madrid y Barcelona. In: Atheneas Digital 12/1, 15-28.

Capel Sáez, Horacio (2007): El debate sobre la construcción de la ciudad y el llamado 'Modelo Barcelona'. In: Revista Electrónica de Geografía y Ciencias Sociales XI/233, 229-255.

Crouch, Colin (2008): Postdemokratie. Frankfurt a. M.: Suhrkamp Verlag.

Cruz i Gallach, Helena (2006): Los conflictos urbanísticos: Sus causas y sus protagonistas. Una reflexión a partir de la experiencia de Cataluña. In: Cuaderno de Geografía 80, 183-194.

Cruz i Gallach, Helena/Martí i Costa, Marc (2010): Conflictos urbanísticos y movilizaciones ciudadanas. Reflexiones desde Barcelona. In: Finisterra XLV 90, 111-132.

Einig, Klaus/Grabher, Gernot/Ibert, Oliver/Strubelt, Wendelin (2005): Urban Governance. Eine Einführung. In: Informationen zur Raumentwicklung 9/10: I-IX.

Fernández Sánchez, Noemí (2007): Innovationskultur in der Metropolregion Barcelona. In: Standort 31/4, 184-188.

Garcia-Ramon, Maria-Dolors/Albet, Abel (2000): Pre-Olympic and post-Olympic Barcelona, a 'model' for urban regeneration today? In: Environmental Planning A 32/o8, 1331-1334.

Krasmann, Susanne (2010): Jacques Rancière: Politik und Polizei im Unvernehmen. In: Ulrich Bröckling/Robert Feustel (Hg.), Das Politische Denken. Zeitgenössische Positionen. Bielefeld: transcript Verlag, 77-98.

Kuhn, Armin (2012): Zwischen gesellschaftlicher Intervention und radikaler Nischenpolitik. Häuserkämpfe in Berlin und Barcelona am Übergang zur neoliberalen Stadt. In: Hanno Balz / Jan-Henrik Friedrichs (Hg.), “All we ever wanted ...” Eine Kulturgeschichte europäischer Protestbewegungen der 1980er Jahre. Berlin: Karl Dietz Verlag, 37-52.

Marchart, Oliver (2010): Die politische Differenz. Zum Denken des Politischen bei Nancy, Lefort, Badiou, Laclau und Agamben. Berlin: Suhrkamp Verlag.

Marina Port Vell (2011): The transformation is underway. www.marinaportvell.com/en (letzter Zugriff am 18.10.2013).

Marshall, Tim (2000): Urban planning and governance: Is there a Barcelona model? In: International Planning Studies 5/3, 299-319.

Marshall, Tim (1996): Barcelona-fast forward? City entrepreneurialism in the 1980 s and 1990s. In: European Planning Studies 4/2, 147-165.

Mayer, Margit (2003): The onward sweep of social capital: causes and consequences for understanding cities, communities and urban movements. In: International Journal of Urban and Regional Research 27/1, 110-132.

Meyer, Katrin (2011): Kritik der Postdemokratie. In: Leviathan 39/1, 21-38.

Monclús, Francisco-Javier (2003): The Barcelona model: and an original formula? From 'reconstruction' to strategic urban projects (1979-2004). In: Planning Perspectives 18/4, 399-421.

Mouffe, Chantal (2005): On the Political. London/New York: Routledge.

Pascual Molinas, Núria/Ribera Fumaz, Ramon (2009): Retail gentrification in Ciutat Vella, Barcelona. In: Libby Porter/Kate Shaw (Hg.), Whose Urban Renaissance? An International Comparison of Urban Regeneration Strategies. London/New York: Routledge, 181-190.

Pauné, Meritxell M. (2013): Vecinos y académicos proponen un Port Vell marinero como alternativa a los yates. www.lavanguardia.com/local/barcelona/20130215/54365409278/ port-vell-marinero-alternativa-yates.html (letzter Zugriff am 27.4.2013).

Plataforma Defensem el Port Vell (2012): Manifiesto de la plataforma Defendamos el Port Vell. http://defensemportvell.wordpress.com/tag/manifest (letzter Zugriff am 10.10.2013).

Rancière, Jacques (2008): Zehn Thesen zur Politik. Berlin/Zürich: Diaphanes Verlag.

Rancière, Jacques (2002): Das Unvernehmen. Politik und Philosophie. Frankfurt a. M.: Suhrkamp Verlag.

Rancière, Jacques (1997): Demokratie und Postdemokratie. In: Alain Badiou/Jacques Rancière/Rado Riha/Jelica Sumic-Riha (Hg.), Politik der Wahrheit. Wien: Verlag Turia \& Kant, 94-122.

Renner, Andreas/Gohl, Christopher/de Maizière, Maximilian (2002): Nachhaltigkeit und Globalisierung, Partizipation, Demokratie-Identifizierung von Zusammenhängen und Gestaltungsansätzen. Kurzstudie für den Rat für Nachhaltige Entwicklung. Bensheim/ Berlin. www.reocities.com/utkum/KurzstudieGlobalisierung.pdf (letzter Zugriff am 19.10.2013). 
Schmidt, Manfred G. (2000): Demokratietheorien. Opladen: Leske + Budrich Verlag.

Schnur, Olaf/Drilling, Matthias (2009): Governance - ein neues Zauberwort auch für die Quartiersentwicklung? In: Matthias Drilling/Olaf Schnur (Hg.), Governance der Quartiersentwicklung. Theoretische und praktische Zugänge zu neuen Steuerungsformen. Wiesbaden: VS-Verlag für Sozialwissenschaften, 11-26.

Simons, Maarten/Masschelein, Jan (2010): Governmental, political and pedagogic subjectivation: Foucault with Rancière. In: Educational Philosophy and Theory 42/5-6, 588-605.

Žižek, Slavoj (2010): Die Tücke des Subjekts. Berlin: Suhrkamp Verlag.

\section{The Barcelona model - participation, protest and post-politics}

The 'Barcelona Model' stands for decision-making and development processes where different actors are included in urban planning. Today's highly formalised options for citizen participation are seen as continuation of the neighbourhood resistance in the 1970s. Increasing formalisation of participation has been regarded as a process of democratisation. The article questions the connection between formalised participation options and democracy. For this purpose the traditional understanding of democracy is confronted with an emancipatory understanding of democracy following the theories of poststructuralist political philosophy. Analysing the protests against the reconstruction of Marina Port Vell in Barcelona provides empirical evidence of limitations of formal participation. Following, the article also discusses, if protest can be viewed as an alternative to formal participation. 